\title{
MicroRNA profile of paclitaxel-resistant serous ovarian carcinoma based on formalin-fixed paraffin-embedded samples
}

\author{
Xiao Li $i^{1,2}$, Yaer Lư ${ }^{1,3}$, Yaxia Chen ${ }^{2}$, Weiguo $\mathrm{Lu}^{2}$ and Xing Xie ${ }^{2^{*}}$
}

\begin{abstract}
Background: To assess the feasibility of validating microRNA (miRNA) profile related to paclitaxel-sensitivity in formalin-fixed paraffin-embedded (FFPE) samples of serous ovarian carcinoma (OC) patients.

Methods: Deregulated miRNAs identified by miRNA microarray were further detected in 45 FFPE OC samples using Realtime PCR. Correlations between paired FFPE and frozen tumor samples were analyzed. Survival times were compared between 6 high and low miRNAs groups. Western blot and luciferase reporter assay were used for validating the target of miRNA.

Results: Sixteen up-regulated miRNAs and twenty-three down-regulated miRNAs were revealed in pacilitaxelresistant ST30 cells. The up-regulated miRNAs (miR-320a, 22 and 129-5p) and down-regulated miRNAs (miR-9, 155 and 640) were confirmed in paclitaxel-resistant FFPE tumor samples, compared with paclitaxel-sensitive samples. Higher miR-9 and miR-640 showed better survival time in OC patients. Expressions of miR-9, 155 and 22 in FFPE samples were closely mimicked by those in frozen tissues. RAB34 was validated as a direct target of miR-9.

Conclusions: We validated miRNA profile in pacilitaxel-resistant OC using FFPE samples, which might enable treatment stratification and help us to predict outcomes in OC patients. FFPE samples are feasible materials for miRNA research.
\end{abstract}

Keywords: Ovarian carcinoma, Chemoresistance, Formalin-fixed paraffin-embedded (FFPE), microRNA

\section{Background}

Ovarian carcinoma $(\mathrm{OC})$ is the leading cause of death among patients with gynecologic cancer. Despite initial responsiveness to the chemotherapeutic drugs, most patients eventually develop chemoresistance and succumb to their diseases [1,2]. Drug resistance remains a formidable problem in managing cancer patients. It is clinically important to identify biomarkers that could assist in predicting which patients will respond to chemotherapy, and, which patients will remain refractory to standard treatment.

microRNA (miRNA)s are small noncoding RNAs involved in the initiation and progression of human cancer [3]. As modulators of protein expression they may

\footnotetext{
* Correspondence: xiex@mail.hz.z..cn

2Department of Gynecologic Oncology, Women's Hospital, School of Medicine, Zhejiang University, No.1 Xueshi Road, Hangzhou, Zhejiang 310006, China

Full list of author information is available at the end of the article
}

operate as oncogenes and tumor suppressors [4]. miRNA expression profiles are useful for assessment of the prognosis and chemosensitivity of human cancer. For example, when cisplatin resistance was specifically examined for miRNA expression, 34 miRNAs with statistically significant changes were found in tumors from responders compared with those of non-responders [5]. Among them, Let-7i was the most down-regulated miRNA in the chemo-resistant patients. In another study, elevation of miR-214 was found to be responsible for development of resistance to cisplatin. Blocking miR214 expression enhanced the sensitivity of A2780 cells to cisplatin-induced apoptosis [6]. Eitan et al. also found an array of tumor specific miRNAs associated with response to platinum [7]. However, few studies have focused on tumor miRNA expression patterns associated with paclitaxel-resistance. Several miRNAs are differentially expressed in paclitaxel-resistant and sensitive OC

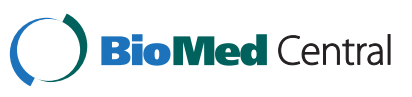


cell lines, when we detected the miRNAs expressions using miRNA microarray in present study. This raises the question that whether a miRNA profile representing paclitaxel-resistance could be useful for prognosticating clinical chemo-resistance in OC patients.

As we know, routine histology processing uses formalin fixation to preserve clinical specimens, which provides a rich resource of tissues linked to clinical databases. In addition, any biomarker developed from formalin-fixed paraffin embedded (FFPE) samples could be more readily translated into clinical practice than frozen tissues. However, most studies of miRNAs still use frozen tissues, despite prospective collection of frozen samples is limited and inefficient $[8,9]$. The most important reason is that RNA is degraded in tissues before, during, and after formalin fixation [10,11]. Interestingly, miRNAs appear to be better preserved, perhaps because of their shorter lengths. Detecting miRNAs using FFPE tissues has been successful for colon, breast and ovarian cancers [12-14]. In present study, we try to validate a panel of miRNAs associated with pacilitaxel-resistant OC using FFPE samples, and to determine that whether certain miRNAs might predict response to chemotherapy and prognosis. Furthermore, to show the suitability of routine FFPE tissue for comprehensive miRNA expression analyses using Realtime PCR, we also compared expressions of miR-9, 155 and 22 in FFPE samples with those in paired frozen samples from the same patients.

\section{Methods}

\section{Patient's characteristics}

FFPE tumor samples $(\mathrm{N}=45)$ were collected in our hospital between Feb 2001 and Mar 2009, from the patients surgically treated for primary serous papillary ovarian adenocarcinoma and received chemotherapy including paclitaxel after primary surgery. Matched frozen samples $(\mathrm{N}=10)$ from the same patients were also obtained (immediately snap-frozen in liquid nitrogen and stored at $-80^{\circ} \mathrm{C}$ ). Only tumor samples with a minimum of $70 \%$ tumor tissue content were included. All pathology slides were evaluated by an expert pathologist. Patients' characteristics are listed in Table 1. Patients with progressive disease during primary chemotherapy or those who suffered recurrent disease within 6 months of completing primary chemotherapy were termed paclitaxel-resistant. Patients with recurrence beyond 6 months or without recurrence were termed paclitaxel-sensitive. Overall survival time (OS) was calculated as the time from surgery to the last follow-up date or death. Progression free survival time (PFS) was calculated as the time from surgery to the time of detected recurrence or progression. The study was approved by the ethical committee of women's hospital, school of medicine, Zhejiang University (Reference number 20070430).
Table 1 Clinical characteristics of 45 ovarian carcinoma patients

\begin{tabular}{cll}
\hline & Sensitive & Resistant \\
\hline Cases number (N) & 24 & 21 \\
Age (range, years) & $52(41-67)$ & $52(32-68)$ \\
FIGO Stage & 3 & 0 \\
I(N) & 1 & 2 \\
II(N) & 18 & 17 \\
III(N) & 2 & 2 \\
IV(N) & & \\
Tumor Grade & 1 & 1 \\
$1(\mathrm{~N})$ & 7 & 2 \\
$2(\mathrm{~N})$ & 16 & 18 \\
$3(\mathrm{~N})$ & & 0 \\
Recurrence & 2 & 21 \\
No (N) & 22 & $6(3-11)$ \\
Yes (N) & $22(12-78)$ & $17(7-65)$ \\
PFS (Range,months) & $42.5(18-144)$ & 20 \\
OS (Range, months) & & 1 \\
Survival & 12 & \\
No (N) & 12 & \\
Yes (N) & & \\
\hline
\end{tabular}

\section{Cell culture and transfection}

Paclitaxel-resistant cell line SKOV3-TR30 (ST30) was induced from SKOV3 (American Type Culture Collection, Manassas, VA, USA). Both were maintained in McCoy's $5 \mathrm{~A}$ medium supplemented with $10 \%$ fetal bovine serum. For ST30 cells, $30 \mathrm{~nm}$ paclitaxel was withdrawn at 2 weeks before experiment. For regulation of miR-9, ST30 cells were transfected with $50 \mathrm{nM}$ miR-9 mimic or its negative Control (Ribobio, GuangZhou, China) by using Lipofectamine 2000 (Invitrogen, Carlsbad, CA, USA) in accordance with the manufacturer's instructions.

\section{RNA extraction and microRNA microarray}

Total RNA was extracted from 45 FFPE tumor samples and 10 FFPE matched frozen tumor samples from the same patients. For snap-frozen tissues, total RNA was isolated by TRIzol(Invitrogen, California, USA). For FFPE tissues, total RNA was extracted by RecoverAll ${ }^{\text {Tn }}$ Total Nucleic Acid Isolation Kit (Ambion, Austin, TX, USA), according to the manufacturer's instructions. Briefly, eight 10-um-thick sections of FFPE samples were deparaffinized with xylene and washed in ethanol. The tissues were digested with protease and treated with DNase. After washing, total RNA was eluted with distilled water. The quantity and quality of RNA were measured by Nanodrop 2000 thermo scientific spectrophotometer (NanoDrop Technologies, Wilmington, DE, USA). 
miRNA expression profiling of SKOV3 and ST-30 cells were detected using the miRCURY LNA Array (version 11.0) system. RNA samples were labeled with the Exiqon miRCURY ${ }^{\mathrm{TM}} \mathrm{Hy}^{\mathrm{TM}} / \mathrm{Hy}^{\mathrm{TM}}$ Power labeling kit and hybridized on the miRCURY LNA Array (version 11.0) station. Scanning was performed with the Axon GenePix 4000B microarray scanner. GenePix pro version 6.0 was used to read image raw intensity of the image. The ratio of red signal to green signal was calculated after background subtraction and normalization using the global Lowess (Locally Weighted Scatter plot Smoothing) regression algorithm (MIDAS, TIGR Microarray Data Analysis System). Between slides normalization was performed by scale normalization to reduce between-slide variability. The experiments were performed in triplicate and repeated three times. The statistical significance of differentially expressed miRNAs was analyzed by fold change and $t$-test. The threshold value we used to screen differentially expressed miRNAs was a fold change $>1.5$ or $<0.67$.

\section{Realtime RT-PCR validation}

The most up-regulated and down-regulated miRNAs identified by microarray were further validated in 45 FFPE tumor samples using realtime RT-PCR. To corroborate the validity of miRNAs expression of FFPE samples, three miRNAs were detected in 10 matched FFPE and snap-frozen samples from the same patients. Correlations of miRNAs expressions between matched FFPE and frozen tumor samples were analyzed.

RT reactions and realtime PCR were performed according to the manufacturer's protocols (TaKaRa, DaLian, China). All RT reactions, including no-template controls were run in a PTC-200 Peltier Thermal Cycler (MJ Research, Waltham, MA, USA). miRNA levels were quantified with the ABI Prism 7900HT sequence detection system (Applied Biosystems, Fostercity, CA, USA). U6 snRNA served as endogenous control. The primers for miRNAs and U6 were obtained from Ribobio (Ribobio, GuangZhou, China). Comparative realtime PCR was performed in triplicate, including no-template controls. Relative expression was calculated using the 2$\Delta \Delta$ ct method.

\section{Western blotting}

At $72 \mathrm{~h}$ after transfection, total protein extracts from the cells were prepared. Total protein was separated by SDS-PAGE and transferred electrophoretically to a nitrocellulose membrane. The membrane was blocked with $5 \%$ nonfat dried milk in TBST buffer, and then incubated overnight at $4^{\circ} \mathrm{C}$ with primary antibodies against RAB34 (SC-27442, 1:250, Santa Cruz) and GAPDH (sc-25778, 1:1000, Santa Cruz) respectively. Blots were washed with TBST buffer and incubated with secondary antibody. The protein bands were visualized using the EZ-ECL kit (Biological Industries, Kibbutz Beit-Haemek, Israel). GAPDH was used as an endogenous control. The experiments were repeated three times.

\section{Dual luciferase reporter assay}

The 3'-untranslated region (UTR) of RAB34 (379BP) mRNA containing the miR-9 binding site were PCR amplified, and then cloned into the pmiR-RB-REPORT ${ }^{\mathrm{TM}}$ dual luciferase reporter vector (Promega) at the 3 ' end of the coding sequence of renilla luciferase using XhoI and NotI restriction sites. To produce the mutations of the miR-9 targeting site, site-directed Gene Mutagenesis Kit (Beyotime, Jiangsu, China) was used according to the manufacturer's protocol. All inserted or mutated sequences were confirmed by sequencing. The primers and mutation primers are listed in Table 2. All primers were synthesized by Ribobio. Cotransfection of miRNA mimic or its negative control $(50 \mathrm{nM})$ and different reporter vectors $(50 \mathrm{nM})$ to $293 \mathrm{~T}$ cells was performed using Lipofectamine 2000. The luciferase activities were measured at 48 hours after transfection using VICTOR $^{\text {TM }}$ multilabel readers (Perkinelmer, Waltham, MA, USA), and the renilla luciferase activities were normalized to firefly luciferase activities. The experiments were performed in triplicate and repeated three times.

\section{Statistical analysis}

Student's $t$-test was used to assess miRNAs expression in FFPE samples and dual luciferase reporter assay. KaplanMeier survival functions and log-rank test were used to assess progress-free survival and overall survival time based on mean expression levels of 6 miRNAs. Pearson's correlation was calculated to compare the PCR results of FFPE samples with those of paired frozen samples. All statistical analyses were two-sided and performed with SPSS 11.5 software package. P-values less than 0.05 were considered statistically significant.

\section{Results}

Confirmed paclitaxel-resistant miRNA profile using FFPE samples

The miRNA microarray revealed that 39 miRNAs showed more than 1.5 fold deregulation between the pacilitaxel resistant and sensitive cell lines (Figure 1A).

Table $\mathbf{2}$ The sequences of primers in the dual luciferase reporter assay

\begin{tabular}{ll}
\hline Primer & Sequence 5'-3' \\
\hline RAB34-WT-F & CCG CTCGAGGGGCTGAGGAGACTGTTC \\
RAB34-WT-R & GAATGCGGCCGCGCTCGTAACAAAGAAATTTAATGCATAAG \\
RAB34-Mut-F & TTATCCAGTCGATACTGCTGCCTCTTGGTGGCAGTA \\
RAB34-Mut-R & CAGCAGTATCGACTGGATAAAGTCAGTGCAAATGT
\end{tabular}

$W T$ : wild type; $F$ : forward, $R$ : reverse; Mut: mutation. The mutation site in the Mut-primer is bold. 


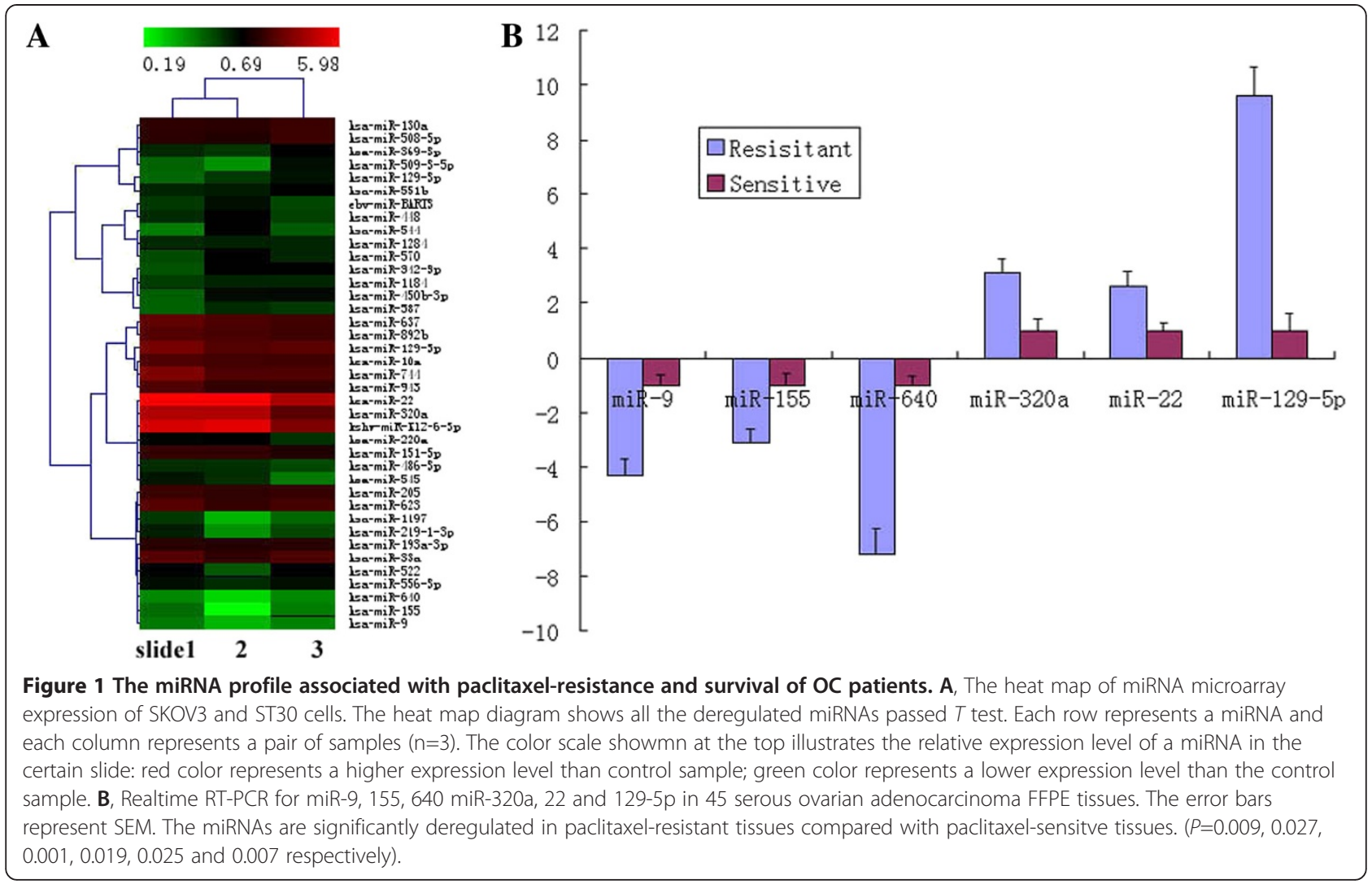

Among them, miR-320a, 22 and 129-5p are the most up-regulated miRNAs, and miR-9, 155 and 640 are the leading down-regulated miRNAs.

To determine the significance of these miRNAs, realtime PCR was performed using 45 FFPE ovarian carcinoma samples. miR-320a, 22 and 129-5p were significantly up-regulated in FFPE paclitaxel-resistant tumor samples $(P=0.019,0.025,0.007)$, while miR-9, 155 and 640 were significantly down-regulated $(P=0.009,0.027$ and 0.001 respectively) (Figure $1 \mathrm{~B}$ ), compared with paclitaxel-sensitive samples. Furthermore, we divided all tissues into high and low miRNAs expression groups based on the mean values of 6 miRNAs, and found longer PFS (21.25 months, $P=0.027)$ and OS (62.63 months, $P=0.021)$ in patients with high miR-9 expression than those with low miR-9 level (12.51 months and 30.40 months, respectively) (Figure $2 \mathrm{~A}$ and $\mathrm{B}$ ). Higher miR640 showed significantly longer OS (69.38 months compared with 35.12 months, $P=0.043$, Figure 2F). Higher miR-155 and 640, lower 320a, 22 and 129-5p showed higher PFS while higher-155 showed longer OS, although not significantly (Figure 2C-E, G, I and K). This data validates a miRNA profile that is characteristically expressed in paclitaxel-resistant $\mathrm{OC}$ tissues and suggests poorer prognosis for $\mathrm{OC}$ patients.

\section{Consistent miRNA expressions validated between paired frozen and FFPE samples}

To further corroborate the validity of miRNA profile of FFPE samples, realtime RT-PCR were repeated using pairs of frozen and FFPE tissues from the same patients. Correlation analysis of miR-9, 155 and 22 levels in FFPE and frozen samples from the same patients showed significant correlationship of the paired samples, with Pearson's rho of $0.88,0.89$ and 0.87 respectively (all $P=0.001$ ). Thus, miRNA expression studies can be reliably performed with routinely obtained pathological materials and the results are similar to the yield from snap-frozen tissues.

\section{RAB34 is one of the targets directly regulated by of miR-9}

The relationship between miRNAs level and prognosis of $\mathrm{OC}$ patients triggered us to investigate the biological consequence of altered miR-9. We predicted some gene targets of miR-9 involved with important cellular signal pathways using TargetScan database (www.targetscan. org, Released 6.0, Nov 2011). RAB34 contained the putative miR-9 binding site, indicating that it might be one of the targets regulated by miR-9. Western blot analysis showed that overexpression of miR-9 by specific mimic, down-regulated the expression of RAB34 in ST30 cells (Figure 3A). Using dual luciferase reporter assay, we 

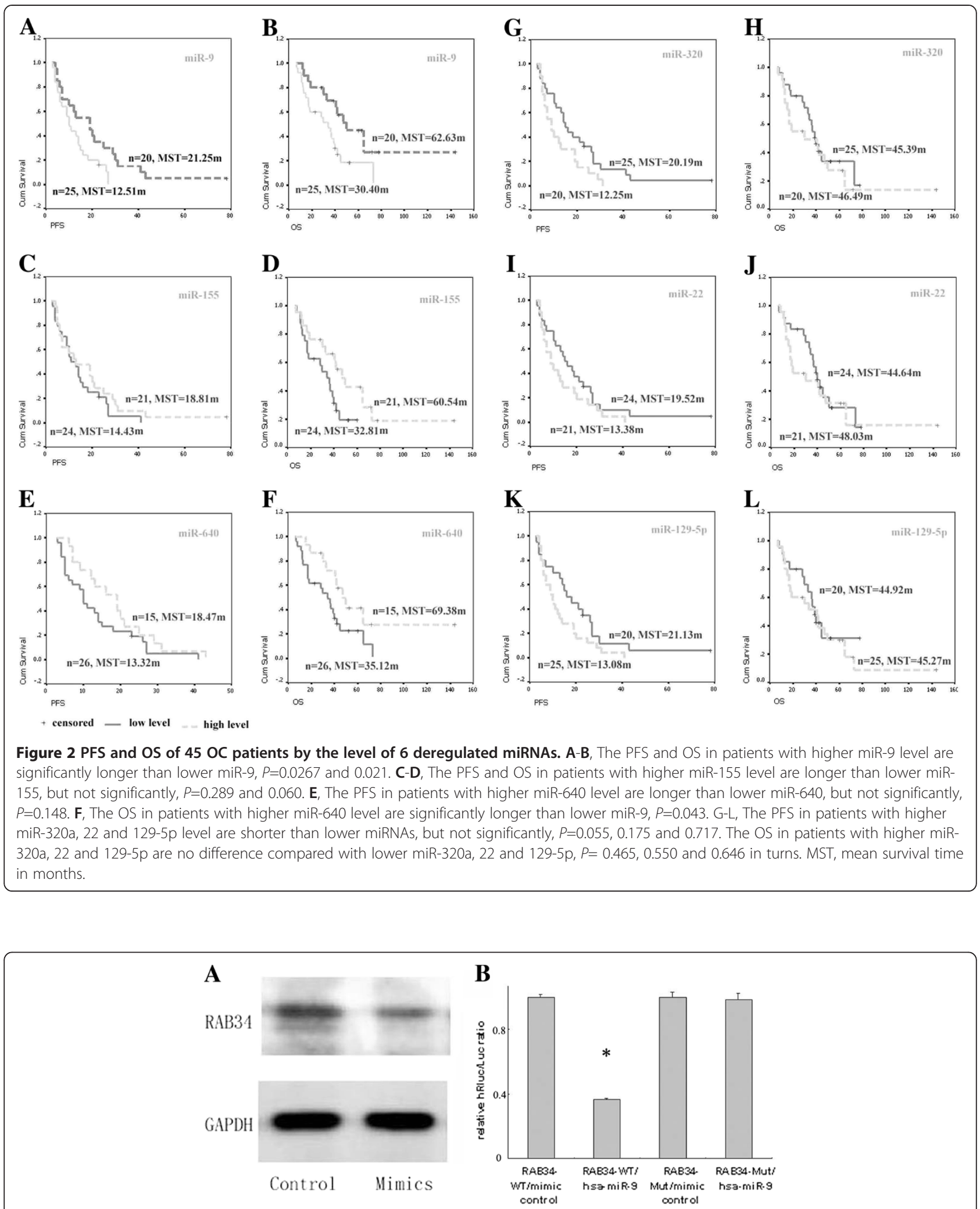

Figure 3 RAB34 is the direct target of miR-9. A, Western blot analysis of RAB34 in ST30 cells transfected with miR-9 mimic or negative control. GAPDH was used as house-keeping gene. B, Dual luciferase reporter assay. 293T cells were transfected with RAB34-wild type 3'UTR vectors or mutant 3'UTR vectors together with miR-9 mimic or its negative control. Luciferase activity was measured $48 \mathrm{~h}$ after co-transfection, and the error bars represent SD. A decrease of the luciferase activity was observed in miR-9 over-expressing cells (* $P=0.000$ ). 
further found that the relative luciferase activities were significantly reduced in cells transfected with RAB34 WT-3'UTR/mirR-9 mimic vectors compared with those transfected with RAB34 WT-3'UTR/mirR-9 mimic control $(P=0.000)$, but the relative luciferase activities were not altered in cells transfected with RAB34 Mut-3'UTR/ miR-9 mimic or RAB34 Mut-3'UTR/miR-9 mimic control (Figure 3B). Collectively, these results validate that miR-9 binds directly to the sites within the 3'UTR of RAB34 and RAB34 is the direct target of miR-9.

\section{Discussion}

Despite multiple new approaches to treatment, the high death rates from OC remain largely unchanged with a 5year overall survival of only $30-39 \%$ [15]. Dramatically poor prognosis of OC patients is due to the development of chemo-resistance. As shown in Table 1, the median PFS in paclitaxel-sensitive group is 22 months, significantly longer than that in paclitaxel-resistant group (6 months, $P=0.000$ ), and the median OS in paclitaxel-sensitive group is also significantly longer than that in paclitaxel-resistant group ( 42.5 vs 17 months, $P=0.000$ ). Therefore, identifying patients who can benefit from the chemotherapy will have significant clinical implications.

miRNA represents a new class of small non-coding RNA that regulates multiple gene expression. During tumorigenesis and tumor progression, up-regulated miRNA could potentially target and down-regulate tumor suppressor genes, whereas down-regulated miRNA could potentially increase the expression of oncogenes [16]. Increasing evidence supports that miRNA may influence the response of ovarian cancer to chemotherapy [17-19]. However, all these evaluations used frozen tissues. In present study, we provide the first characterization of the paclitaxel-resistant miRNA profiling of $45 \mathrm{FFPE}$ samples from primary serous papillary ovarian adenocarcinoma patients using realtime PCR assay. Three top deregulated miRNAs were selected for validation using 10 paired FFPE and frozen samples from the same patients. Importantly, the changes predicted for these 3 miRNAs using the paired samples from the same patients were significantly correlated. Thus, these data demonstrate that FFPE samples may be used as a feasible material for miRNA expression research. Although different types of tumors may have the same miRNAs markers, there are specific miRNAs in tumors from different cellular origins [20]. In present study, the majority of the differentially expressive miRNAs have not been reported before, such as miR-9 and miR-640. This discrepancy may be attributed to different patient population and inter-experimental variations. Our study focused on primary serous papillary ovarian adenocarcinoma patients and paclitaxel-sensitivity, whereas previous studies were involved different histological types of ovarian cancer and chemotherapy agents [5-7].
Specific data may assist in tailoring treatment to each patient's specific clinical situation during the initial management of their disease and also offer the opportunity for better counseling regarding prognosis. Based on the confirmed top deregulated miRNAs in paclitaxel-resistant ovarian carcinoma, we further analyzed the associations of different miRNAs expression and survival outcomes after stratifying the OC patients into high and low miRNAs' levels. Our analyses showed that patients those with high miR-9 and 640 level survived better than those with low results. Moreover, deregulation of miR-155, 320a, 22 and 129-5p were found to be associated with shorter PFS of the patients, although not significantly. Thus, our findings suggest that a miRNA profile may help us to distinguish patients who will respond to paclitaxel treatment and provide biomarker of poor prognosis in OC patients. However, given the small number of patients involved in the study, these results need to be interpreted with caution.

Understanding the regulatory role of miRNA may lead to better understanding of the molecular events involved in different biological processes, and,lead to the development of diagnostic tools and a novel class of drug targets for therapeutic interventions. According to the TargetScan database, there are over 1200 predicted hsa-miR- 9 targets, including some familiar oncogenes. Among them, RAB34 has been confirmed as a direct target of miR-9 in OC using western blot and dual luciferase reporter assay. As a member of RAS oncogene family, RAB34 is a guanosine triphosphatase (GTPases) that can regulate budding junction and fusion of vesicle in exocytosis and endocytosis pathway [21]. Luo et al. [22] has found that miR-9 was down-regulated and can be the modulator of RAB34 in gastric carcinoma, which was concordant with our finding.

\section{Conclusions}

There is still work to be done for better defining the mechanisms of miRNA-associated paclitaxel resistance and its potential as a future target for therapy in OC. However, our results support the conclusions that miRNA profile allows meaningful subclassification of paclitaxelresistant ovarian tumors and prognosis prediction. FFPE samples are convenient and feasible materials for miRNA study. In addition, we find miR-9 might be correlated with the paclitaxel-sensitivity through targeting RAB34. Hopefully, these findings can ultimately be used to improve ovarian cancer patient outcome.

Competing interests

The authors declare that they have no competing interests.

\section{Authors' contributions}

$\mathrm{XL}$ carried out cell culture, $\mathrm{PCR}$, Western Blot and drafted the manuscript. YL and YC collected the patient's materials and followed up. WL carried out Dual luciferase reporter assay. XX conceived the study and revised the manuscript. All authors read and approved the final manuscript. 


\section{Acknowledgements}

This work was funded by the project of Zhejiang Provincial Natural Science Foundation of China (Grant No.Y207054), National Natural Science Foundation of China (Grant No.81001164), the Fundamental Research Funds for the Central Universities (2010), and National High Technology Research and Development Program (863) (2012AA02A507).

\section{Author details}

'Women's Reproductive Health Laboratory of Zhejiang Province, Women's Hospital, School of Medicine, Zhejiang University, Hangzhou, China. 2Department of Gynecologic Oncology, Women's Hospital, School of Medicine, Zhejiang University, No.1 Xueshi Road, Hangzhou, Zhejiang 310006, China. ${ }^{3}$ Zhejiang Provincial People's Hospital, Hangzhou, Zhejiang, China

Received: 27 November 2012 Accepted: 25 April 2013

Published: 30 April 2013

\section{References}

1. Jemal A, Siegel R, Ward E, Hao Y, Xu J, Murray T, Thun MJ: Cancer statistics, 2008. CA Cancer J Clin 2008, 58:71-96

2. Kyrgiou M, Salanti G, Pavlidis N, Paraskevaidis E, loannidis JP: Survival benefits with diverse chemotherapy regimens for ovarian cancer: metaanalysis of multiple treatments. J Natl Cancer Inst 2006, 98:1655-1663.

3. Garzon R, Calin GA, Croce CM: MicroRNAs in cancer. Annu Rev Med 2009, 60:167-179.

4. Chen CZ: MicroRNAs as oncogenes and tumor suppressors. N Engl J Med 2005, 353:1768-1771.

5. Yang N, Kaur S, Volinia S, Greshock J, Lassus H, Hasegawa K, Liang S, Leminen A, Deng S, Smith L, Johnstone CN, Chen XM, Liu CG, Huang Q, Katsaros D, Calin GA, Weber BL, Bützow R, Croce CM, Coukos G, Zhang L: MicroRNA microarray identifies Let-7i as a novel biomarker and therapeutic target in human epithelial ovarian cancer. Cancer Res 2008, 68:10307-10314

6. Yang H, Kong W, He L, Zhao JJ, O'Donnell JD, Wang J, Wenham RM, Coppola D, Kruk PA, Nicosia SV, Cheng JQ: MicroRNA expression profiling in human ovarian cancer: miR-214 induces cell survival and cisplatin resistance by targeting PTEN. Cancer Res 2008, 68:425-433.

7. Eitan R, Kushnir M, Lithwick-Yanai G, David MB, Hoshen M, Glezerman M, Hod M, Sabah G, Rosenwald S, Levavi H: Tumor microRNA expression patterns associated with resistance to platinum based chemotherapy and survival in ovarian cancer patients. Gynecol Oncol 2009, 114:253-259.

8. Gillis A, Stoop H, Hersmus R, Oosterhuis JW, Sun Y, Chen C, Guenther S, Sherlock J, Veltman I, Baeten J, van der Spek PJ, de Alarcon P, Looijenga LH: High- throughput microRNAome analysis in human germ cell tumours. J Pathol 2007, 213:319-328.

9. Gramantieri L, Ferracin M, Fornari F, Veronese A, Sabbioni S, Liu CG, Calin GA, Giovannini C, Ferrazzi E, Grazi GL, Croce CM, Bolondi L, Negrini M: Cyclin G1 is a target of miR-122a, a microRNA frequently downregulated in human hepatocellular carcinoma. Cancer Res 2007, 67:6092-6099.

10. Macabeo-Ong M, Ginzinger DG, Dekker N, McMillan A, Regezi JA, Wong DT, Jordan RC: Effect of duration of fixation on quantitative reverse transcription polymerase chain reaction analyses. Mod Pathol 2002 15:979-987.

11. Bresters D, Schipper ME, Reesink HW, Boeser-Nunnink BD, Cuypers HT: The duration of fixation influences the yield of HCV CDNA-PCR products from formalin-fixed, paraffin-embedded liver tissue. J Virol Methods 1994, 48:267-272.

12. Xi Y, Nakajima G, Gavin E, Morris CG, Kudo K, Hayashi K, Ju J: Systematic analysis of microRNA expression of RNA extracted from fresh frozen and formalin-fixed paraffin-embedded samples. RNA 2007, 13:1668-1674.

13. Sempere LF, Christensen M, Silahtaroglu A, Bak M, Heath CV, Schwartz G, Wells W, Kauppinen S, Cole CN: Altered microRNA expression confined to specific epithelial cell subpopulations in breast cancer. Cancer Res 2007 67:11612-11620.

14. Laios A, OToole S, Flavin R, Martin C, Kelly L, Ring M, Finn SP, Barrett C, Loda M, Gleeson N, D'Arcy T, McGuinness E, Sheils O, Sheppard B, O'Leary J: Potential role of miR-9 and miR-223 in recurrent ovarian cancer. Mol Cancer $2008,7: 35$
15. Parmar MK, Ledermann JA, Colombo N, du Bois A, Delaloye JF, Kristensen GB, Wheeler S, Swart AM, Qian W, Torri V, Floriani I, Jayson G, Lamont A, Tropé C: ICON and AGO Collaborators. Paclitaxel plus platinum-based chemotherapy versus conventional platinum- based chemotherapy in women with relapsed ovarian cancer: the ICON4/AGO -OVAR-2.2 trial. Lancet 2003, 361:2099-2106.

16. Liu S, Kumar SM, Lu H, Liu A, Yang R, Pushparajan A, Guo W, Xu X: MicroRNA-9 up-regulates E-cadherin through inhibition of NF-KB1-Snail1 pathway in melanoma. J Pathol 2012, 226:61-72.

17. Helleman J, Jansen MP, Burger C, van der Burg ME, Berns EM: Integrated genomics of chemotherapy resistant ovarian cancer: a role for extracellular matrix, TGF beta and regulating microRNAs. Int J Biochem Cell Biol 2010, 42:25-30.

18. van Jaarsveld MT, Helleman J, Berns EM, Wiemer EA: MicroRNAs in ovarian cancer biology and therapy resistance. Int J Biochem Cell Biol 2010, 42:1282-1290

19. Sorrentino A, Liu CG, Addario A, Peschle C, Scambia G, Ferlini C: Role of microRNAs in drug-resistant ovarian cancer cells. Gynecol Oncol 2008, 111:478-486.

20. Barad O, Meiri E, Avniel A, Aharonov R, Barzilai A, Bentwich I, Einav U, Gilad S, Hurban P, Karov Y, Lobenhofer EK, Sharon E, Shiboleth YM, Shtutman M, Bentwich Z, Einat P: MicroRNA expression detected by oligonucleotide microarrays system establishment and expression profiling in human tissues. Genome Res 2004, 14:2486-2494.

21. Chen T, Han Y, Yang M, Zhang W, Li N, Wan T, Guo J, Cao X: Rab39, a novel Golgi-associated Rab GTPase from human dendritic cells involved in cellular endocytosis. Biochem Biophys Res Commun 2003, 303:1114-1120.

22. Luo H, Zhang H, Zhang Z, Zhang X, Ning B, Guo J, Nie N, Liu B, Wu X: Down-regulated miR-9 and miR-433 in human gastric carcinoma. J Exp Clin Cancer Res 2009, 28:82.

doi:10.1186/1471-2407-13-216

Cite this article as: Li et al:: MicroRNA profile of paclitaxel-resistant serous ovarian carcinoma based on formalin-fixed paraffin-embedded samples. BMC Cancer 2013 13:216.

\section{Submit your next manuscript to BioMed Central and take full advantage of:}

- Convenient online submission

- Thorough peer review

- No space constraints or color figure charges

- Immediate publication on acceptance

- Inclusion in PubMed, CAS, Scopus and Google Scholar

- Research which is freely available for redistribution 